\title{
Estudos para implantação de ferramenta de apoio à gestão de linguagens documentárias: vocabulário controlado da USP'
}

\section{Studies to implement an indexing language management tool: the USP controlled vocabulary}

\author{
Vânia Mara Alves de LIMA² \\ Nair Yumiko KOBASHI ${ }^{3}$ \\ Mariza Leal de Meirelles do COUTTO 4 \\ Cibele Araujo Camargo Marques dos SANTOS ${ }^{5}$ \\ Maria Célia AMARAL ${ }^{6}$ \\ Sandra TOKAREVICZ7 \\ Silvia Regina Saran Della TORRE ${ }^{8}$ \\ Sonia Regina Yole GUERRA ${ }^{8}$ \\ Vera Regina Casari BOCCATO 9 \\ João Carlos Holland BARCELLOS ${ }^{10}$
}

\section{RESUMO}

A qualidade dos recursos informacionais armazenados em Bases de Dados depende, em larga medida, dos padrões utilizados em seu tratamento. Nesse contexto, ganha importância primordial a linguagem de indexação do sistema. O vocabulário controlado utilizado na indexação e recuperação de informação requer atualização contínua, baseada em métodos rigorosos, que contemplem o trabalho coletivo dos integrantes do sistema e a linguagem do usuário. Apresentam-se neste trabalho os estudos realizados pelo Grupo de Gerenciamento do Vocabulário Controlado do Sistema Integrado de Bibliotecas da Universidade de São Paulo para a implantação de estrutura e instrumentos específicos de gestão do vocabulário. A ferramenta de apoio à gestão é um aplicativo constituído de um formulário online com as seguintes funcionalidades: solicitação de

1 Trabalho divulgado sob a forma de pôster e resumo no XXI CBBD - Congresso Brasileiro de Biblioteconomia e Documentação, Curitiba, PR, 2005.

2 Coordenadora de Conteúdo (Bibliotecária), Faculdade de Arquitetura e Urbanismo/Biblioteca. Universidade de São Paulo. São Paulo, SP, Brasil.

3 Assessora da Coordenação de Conteúdo, Professora Doutora, Escola de Comunicação e Artes, Universidade de São Paulo. São Paulo, SP, Brasil.

4 Coordenadora de Processo (Bibliotecária), SIBi, Departamento Técnico, Universidade de São Paulo. São Paulo, SP, Brasil.

5 Grupo Manutenção em Ciências Biológicas (Bibliotecária), Faculdade de Saúde Pública, Universidade de São Paulo. São Paulo, SP, Brasil.

- Grupo Manutenção em Ciências Humanas (Bibliotecária), Faculdade de Filosofia, Letras e Ciências Humanas, Universidade de São Paulo. São Paulo, SP, Brasil.

7 Grupo Manutenção em Ciências Humanas (Bibliotecária), Escola de Comunicação e Artes, Universidade de São Paulo. São Paulo, SP, Brasil.

8 Grupo Manutenção em Ciências Exatas e Tecnológicas (Bibliotecárias). Universidade de São Paulo. São Paulo, SP, Brasil.

- Grupo Manutenção em Ciências Biológicas (Bibliotecária), FOB, Universidade de São Paulo. Alameda Octávio Pinheiro Brisolla, 9-75, Vila Universitária, 17012-901, Bauru, SP, Brasil. Correspondência para/Correpondence to: V.R.C. BOCCATO. E-mail: <vboccato@fob.usp.br>.

10 Coordenação de Processos (Analista de Sistemas), SiBi, Departamento Técnico Universidade de São Paulo. São Paulo, SP, Brasil. Recebido em 8/9/2005 e aceito para publicação em 1/12/2005. 
inclusão, modificação, exclusão de termos (descritor ou remissiva) e controle das diversas instâncias de validação. O fluxo da ferramenta tem início com a proposição de modificações pelas bibliotecas base do sistema, e passa, em seguida, por dois filtros de validação: a) grupo de manutenção do vocabulário controlado, constituído por representantes das três grandes áreas do conhecimento, que compõe a macroestrutura do vocabulário; b) grupo de coordenação de conteúdo. Validadas as propostas, passa-se à inclusão do termo no vocabulário controlado e à autorização para seu uso. A ferramenta, criada pelo Departamento Técnico do Sistema de Bibliotecas da USP, com base nos procedimentos de gestão adotados pelo Grupo de Gerenciamento do Vocabulário, caracteriza-se como ferramenta de trabalho em rede. Espera-se que esta ferramenta promova um maior controle de qualidade do vocabulário, otimize os processos de tratamento da informação e redunde em benefícios crescentes para os usuários da base DEDALUS.

Palavras-chave: vocabulário controlado; sistema de gestão; banco de dados.

\section{A B STR A C T}

The quality of the information resources stored in databases greatly depends upon the standards used in their processing. In this context, an adequate indexing language is essential. The controlled vocabulary used in indexing and information recovery requires continuous updating based on rigid standards, which contemplate the collective work of the system participants and the user's language. This work introduces the studies performed by the 'Controlled Vocabulary Management Group of the Integrated System of Libraries of the University of São Paulo for implementing structure and specific tools for vocabulary management. The tool which supports management is a software package with the following functionalities: a request for inclusion modification or exclusion of terms (descriptor or non-descriptor) and a validating process control. The tool flow begins with a proposal for demands throughout the basic system libraries and goes on through two validation filters which consist of: a) a maintenance group for controlled vocabulary, composed of representatives of the three knowledge areas that make up vocabulary macrostructure; b) a coordination group for content. After the validation process, the vocabulary is updated and the use of new terms is authorized. This tool was designed by the Technical Department of USP System of Libraries, and consists of a network instrument to promote a higher quality control of the vocabulary. The main goal is to optimize information retrieval, and to increase the benefits for DEDALUS Database users.

Key words: controlled vocabulary; management system; database.

\section{N T R O D U Ç Ã O}

A organização e a recuperação da informação fundamentam-se em pressupostos largamente aceitos na área da Ciência da Informação. O pressuposto mais importante a ser destacado é o que afirma que a organização da informação é uma atividade intelectual que apresenta, ao menos, três dimensões (SVENONIUS, 2001):

a) dimensão social: se explicita no fato de ser uma atividade institucional; nessa medida, ela deve estar em harmonia com os objetivos da instituição no interior da qual se desenvolve;

b) dimensão teórico/metodológica: se relaciona aos aspectos cognitivos, ou seja, ao conhecimento crítico acumulado pela área, o que permite propor hipóteses de solução de problemas do campo e

c) dimensão operacional: que se refere ao modo de organizar o trabalho, tendo em vista os instrumentos que podem ser utilizados no processo. Esta dimensão é de natureza técnica e tecnológica.

A trajetória percorrida para a criação do "Vocabulário Controlado do Departamento Técnico do Sistema de Bibliotecas da USP (SIBi/USP)", que pode ser descrita em duas etapas, responde às dimensões acima mencionadas. A primeira etapa resultou do desenvolvimento de um projeto sistêmico do Sistema Integrado de Bibliotecas da Universidade de São Paulo (SIBi/USP), do qual participaram os profissionais das Bibliotecas da Universidade, o que caracteriza a dimensão social do projeto; contou com pesquisadores/docentes dos cursos de Biblioteconomia da USP e Universidade Estadual Paulista (UNESP) que foram responsáveis pela orientação metodológica; recorreu, além disso, a professores e especialistas nos assuntos integrados ao Vocabulário do SIBi/USP, para garantir a consistência da terminologia e dos sistemas conceituais de cada área do conhecimento, caracterizando a sua dimensão 
teórico-metodológica. A dimensão operacional se expressa nas atividades de suporte da área da informática que tornou factível a execução do projeto. Inicialmente veiculado em CD-Rom, o resultado do projeto, em forma de base de dados, foi implementado para uso pelo Banco de Dados Bibliográficos da USP (Dedalus), catálogo on-line das Bibliotecas do SIBi/USP, agregando valor ao mesmo. No momento, encontra-se disponível para acesso on-line, ao público em geral, a partir do endereço web <http://www.usp.br/sibi>.

Com o lançamento do produto "Vocabulário Controlado do SIBi/USP", encerrou-se o primeiro projeto, que pode ser acessado pelo endereço <http:/ /www.sibi.usp.br/gestao> Passou-se, a seguir, à elaboração de um segundo projeto para definir o modelo de manutenção e gerenciamento do Vocabulário. Esta etapa de trabalho, referente à implantação e aos primeiros passos da manutenção do vocabulário controlado, foi descrita em trabalho anterior (KOBASHI; LIMA, 2004).

O modelo de gerenciamento ora apresentado (Anexo 1) decorreu de recomendações constantes do relatório final do segundo projeto. O modelo define os objetivos do Grupo de gerenciamento, sua estrutura e responsabilidades dos membros. Essa proposta foi validada, institucionalizada e, dessa forma, incorporada aos processos sistêmicos de responsabilidade do Departamento Técnico do SIBI/USP. Pela sua peculiaridade, envolve um Grupo de Gerenciamento, constituído por bibliotecários do SIBi/USP e técnicos de seu Departamento Técnico, como também por especialista em Linguagens Documentárias da área acadêmica - Departamento de Biblioteconomia da Escola de Comunicações e Artes da USP (ECA/CBD) (Anexo 2). A indicação de membros do Grupo é feita a cada dois anos, procurando-se, com essa medida, renovar o grupo, de modo a garantir a continuidade dos trabalhos e com incorporação de novos membros.

O processo de manutenção e aprimoramento do "Vocabulário Controlado do SIBi/USP" demanda participação de todas as Bibliotecas do Sistema, das quais se espera a apresentação de novas sugestões baseadas em estudos da terminologia das áreas específicas do conhecimento. Requer, também, intenso trabalho de desenvolvimento de aplicativos para dar suporte ao gerenciamento do Vocabulário.
A presente etapa de trabalho teve início em dezembro de 2004, em ambiente favorecido pelas TICs - Tecnologias de Informação e Comunicação. Com efeito, as TICs vêm modificando profundamente os processos de trabalho, em particular os de natureza intelectual, que requerem trabalho cooperativo em rede. Semelhante reorganização de processos tem recebido novas denominações, tal como a de networking (BELLUZZO, 2003).

Muitos autores têm analisado e discutido o novo contexto social e econômico em que nos encontramos, atribuindo a ele diferentes termos: Era da Informação, Era do Conhecimento, Sociedade da Informação, Sociedade do Conhecimento, entre outros. Embora as perspectivas teóricas dos autores não sejam similares, eles concordam com o fato de que a informação transformou-se em mercadoria da maior importância no mundo contemporâneo (BENNIS, 1999).

Via de regra, os autores também concordam com a idéia de que as tecnologias baseadas nos padrões da internet facilitam imensamente tanto a troca de informações entre as organizações, como aumentam as possibilidades de colaboração em modos síncronos ou assíncronos, independentemente de localização física. A Era da Informação permitiu, ainda, que fossem automatizadas tarefas repetitivas, conferindo maior eficiência e rapidez aos processos de trabalho.

AEra das Redes, no entanto, requer discussão crítica dos novos padrões de sociabilidade (CASTELLS, 2001), pois, de acordo com este autor e outros, a internet está mudando os padrões de interação social, criando uma sociedade caracterizada por um maior individualismo em rede. A rede propicia um aumento significativo das possibilidades de aprendizagem dos indivíduos em razão da liberdade e capacidade de eles criarem suas próprias redes e se comunicarem com número muito maior de pessoas, independentemente de sua localização (TERRA; GORDON, 2002). Por outro lado, as tecnologias da informação e as relações entre redes que, segundo Castells, são processos que predominam na Era da Informação, provocam efeitos contraditórios, já que incluem e excluem, simultaneamente. Inclusão e exclusão são, por sua vez, determinados pelas competências dos participantes para se comunicarem na rede, processo que depende do domínio e compartilhamento de códigos (CASTELLS, 2001). 
O trabalho cooperativo em rede, ao atingir as dimensões pessoal, profissional, organizacional e de oportunidade, amplia as condições para promover a proteção, a segurança, o compartilhamento e a distribuição de informações. São esses aspectos que, se adotados de forma crítica, podem beneficiar os processos coletivos de trabalho. No trabalho do Vocabulário Controlado da USP, em todas as etapas, tem-se procurado dimensionar adequa-damente as relações entre ética, conhecimento e métodos de trabalho para que os recursos tecnológicos beneficiem as atividades da equipe. Além disso, por meio de metodologias rigorosas de trabalho, apoiadas nas TICs, tem sido possível melhorar o compartilhamento de informações, redimensionar atividades, cultivar e manter ativa uma rede de relacionamentos humanos. Pode-se afirmar, portanto, que, neste caso, o capital científico e cognitivo da Universidade (definido como conjunto de habilidades coletivas e pessoais para a consecução de objetivos) vem sendo mobilizado de forma produtiva e multifuncional.

\section{GERENCIAMENTODO VOCABULÁRIO CONTROLADO DO SIBI/USP}

O "Vocabulário Controlado do SIBi/USP" foi implantado para uso pelo Banco DEDALUS, em outubro de 2001, e divulgado em suporte CD-Rom. Finalizada, portanto, a etapa do projeto de criação do Vocabulário, o grupo de trabalho deu início à elaboração de uma proposta que pudesse garantir seu crescimento controlado e harmonioso.

A atualização de um vocabulário controlado é tarefa permanente. Torna-se necessário criar novos descritores, estabelecer novas relações, substituir, corrigir ou suprimir descritores e introduzir notas para descritores ambíguos (GUINCHAT; MENOU, 1994). É oportuno lembrar que muitos sistemas de informação vêm investindo pesadamente em TICs sem, no entanto, fazer o correspondente investimento no tratamento da informação e na permanente avaliação e atualização da indexação e das linguagens utilizadas nesses sistemas.

A política de manutenção e atualização de uma linguagem documentária, na prática, deve prever dois aspectos: o gerenciamento do processo e o controle terminológico.
O primeiro aspecto inclui a elaboração de diretrizes, a definição dos mecanismos de revisão e atualização, o estabelecimento de prazos, a verificação de falhas e solução dos problemas detectados, bem como a comunicação das atualizações executadas a todos os integrantes do sistema (indexadores e usuários, incluídos neste último grupo o setor de referência e o usuário final).

O segundo aspecto refere-se à análise dos descritores e não-descritores, à revisão/atualização das relações entre eles e à consulta de fontes e/ou especialistas da área para garantir a pertinência das modificações a serem introduzidas (SANTOS, 2002).

Este segundo aspecto responde aos princípios da garantia literária e garantia de uso, previstos nas diretrizes e normas de construção e manutenção de vocabulários controlados. Para atendê-los, o "Vocabulário Controlado do SIBi/USP" dispõe de três níveis de controle em seu gerenciamento, cada um deles executado por grupos especialmente criados para essa finalidade. São eles: Grupo de Manutenção, Coordenação de Conteúdo e Coordenação de Processo. A partir do trabalho cooperativo de apresentação de sugestões, pelas 40 Bibliotecas do Sistema, o Grupo de Manutenção e a Coordenação de Conteúdo atuam no controle terminológico. O terceiro nível de controle é de responsabilidade da Coordenação do Processo, que executa as decisões tomadas, ou seja, atualiza a base de dados do Vocabulário.

Observa-se que as atividades de trabalho incluem a dimensão social (que pauta as atividades da Coordenação de Conteúdo), a dimensão cognitiva (desenvolvida majoritariamente pelo Grupo de Manutenção) e a dimensão operacional (realizada fundamentalmente pela Coordenação do Processo).

Um aspecto fundamental a ser considerado no processo de manutenção e gerenciamento do Vocabulário é a qualidade da equipe de trabalho. Isto é, os bibliotecários do Sistema, pesquisadores e técnicos devem compreender o papel da Universidade e estar comprometidos com sua missão. Além disso, deve estar organizada em instâncias que se comunicam permanentemente. Assim, a estrutura atual apresenta as instâncias que se seguem:

- Grupo de Bibliotecas: formado por bibliotecários das 40 bibliotecas do Sistema Integrado de Bibliotecas da Universidade de São Paulo. 
- Grupo de Manutenção: formado por duas bibliotecárias representantes de cada área de conhecimento, incluídas no Vocabulário (Ciências Humanas, Ciências Exatas e Ciências Biológicas), totalizando seis pessoas.

- Grupo de Coordenação de Conteúdo: formada pelo bibliotecário coordenador do conteúdo e por um docente especializado em Linguagens documentárias;

- Coordenação do Processo: formada por bibliotecários e analistas desistemas do DT/SIBi.

As atribuições de cada uma das instâncias são descritas.

- Grupo de Bibliotecas: sugere a atualização do Vocabulário solicitando inclusão, correção ou exclusão de descritores e/ou qualificadores pelo preenchimento do formulário on-line.

- Grupo de Manutenção: coleta as sugestões por área do conhecimento; analisa as solicitações; consulta especialistas, se necessário; consolida e encaminha a decisão para a instância seguinte.

- Grupo de Coordenação de Conteúdo: verifica a consistência da sugestão; valida o descritor e sua localização na estrutura. Tem por competência, ainda, estabelecer o regimento do grupo e a política de indexação.

- Coordenação de Processo: executa as decisões da instância anterior, com a atualização da base de dados do Vocabulário.

O Grupo de Bibliotecas, integrado por bibliotecários indexadores, é a mola mestra da manutenção do Vocabulário. Este grupo detecta suas insuficiências e propõe as modificações julgadas necessárias, com base nas atividades de indexação e de referência que desenvolve.

No conjunto, as atividades do Grupo de Gerenciamento do Vocabulário estão sinteti-zadas, em forma de fluxo (Figura 1).

SISTEMA DE SUGESTÕES DO VOCABULÁRIO CONTROLADO DA USP-SIBIX 653

A manutenção e gerenciamento do Vocabulário se efetiva com o uso do Sistema de Sugestões do Vocabulário Controlado da SIBi/USP,

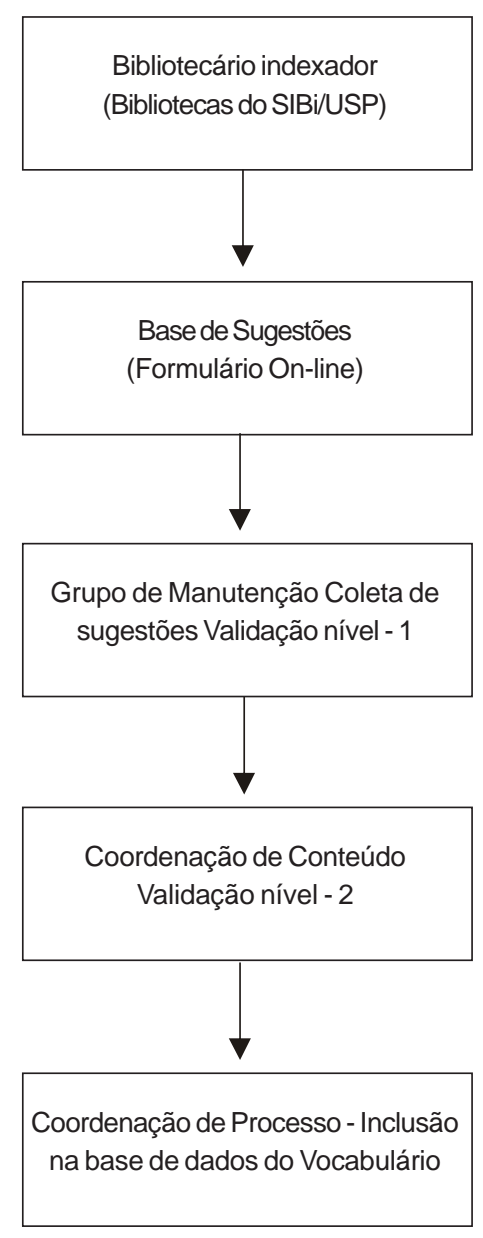

Figura 1. Manutenção e Gerenciamento do Vocabulário Controlado do SIBi/USP: fluxo de Trabalho.

denominado SIBIX 653, desenvolvido pelo Departamento Técnico do SIBi/USP.

O SIBIX 653 foi implementado a partir do delineamento do fluxo de informação proposto pela equipe responsável pelo gerenciamento do Vocabulário. É constituído por uma base de dados, um conjunto de programas que controla o fluxo da informação e propicia a interface para o usuário (bibliotecários indexadores do Sistema), por meio de formulário disponível para preenchimento on-line.

O desenvolvimento da fase inicial (versão 2.0) do sistema foi realizado com requisitos mínimos e suficientes para ocorrer de forma ágil e permitir a rápida disponibilidade para o usuário. O sistema foi desenhado para web e desenvolvido na linguagem PHP, com banco de dados MySQL. Deve ser utilizado em navegadores MS Internet Explorer 5.5 (ou superior) 
ou Netscape versão 6.0 (ou superior). O sistema se encontra dividido em dois módulos principais: o Módulo de Usuários do sistema e o Módulo de Sugestões ou pedidos propriamente ditos.

- Módulo de Usuários do sistema: permite cadastrar usuários, suas respectivas senhas e níveis de segurança. Possui, também, rotinas de listagens/ relatórios e manutenção do cadastro. O nível de segurança estabelece a restrição de acesso às diversas etapas operacionais do sistema.

- Módulo de Sugestões: controla o fluxo do processo de apresentação e validação de sugestões até sua inclusão na base de dados do Vocabulário - SIBIX 650.

O histórico do trâmite do pedido pode ser acompanhado pelo requerente por meio de listagens/ relatórios que mostram a fase em que se encontra o mesmo.

Após os testes nessa etapa e verificado o atendimento pelo sistema de todos os requisitos propostos, está prevista a sua melhoria em relação à interface gráfica para o usuário e a implementação dos dados no formato Dublin Core.

Apresenta-se abaixo a interface, atual com exemplos das várias etapas do fluxo.

A figura 2 apresenta uma solicitação que passou por todas as etapas: análise pelo Grupo de manutenção (validação nível 1), análise pela Coordenação de conteúdo (validação nível 2), e execução pela Coordenação de processo (nível 3), tendo sido aprovada, tal como solicitado pela biblioteca base.

A figura 3 mostra uma solicitação que foi executada, porém, aprovada com alterações pela Coordenação de conteúdo, visando manter a coerência da forma do descritor.

A figura 4 mostra uma solicitação indeferida, com a justificativa da decisão tomada.

$\mathrm{Na}$ figura 5 apresenta-se uma solicitação ainda pendente, porque sua pertinência, ainda, está sendo discutida pela equipe.

O SIBIX 653, por suas funcionalidades, é uma ferramenta essencial de gerenciamento do Vocabulário porque permite fazer o acompanhamento ágil e seguro das decisões tomadas pelas diversas instâncias envolvidas no processo.

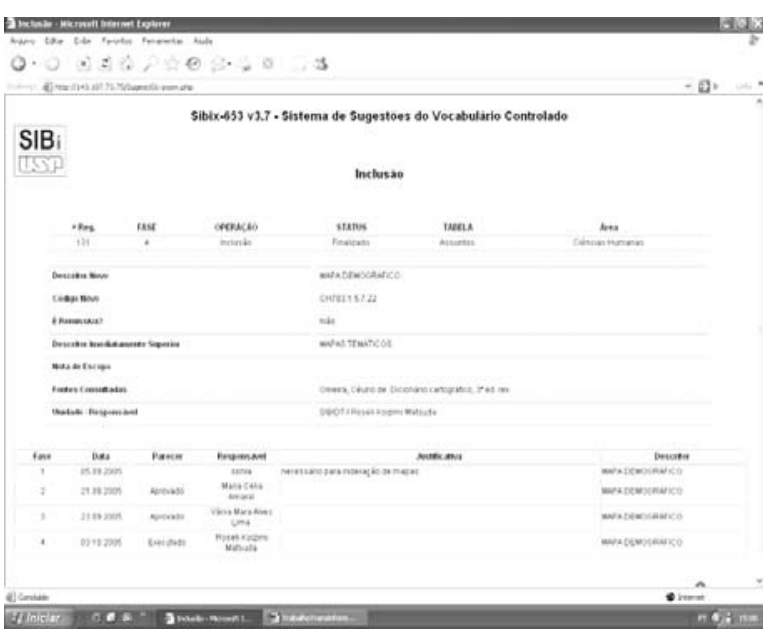

Figura 2. Formulário de Sugestões: solicitação validada pelos três níveis de análise.

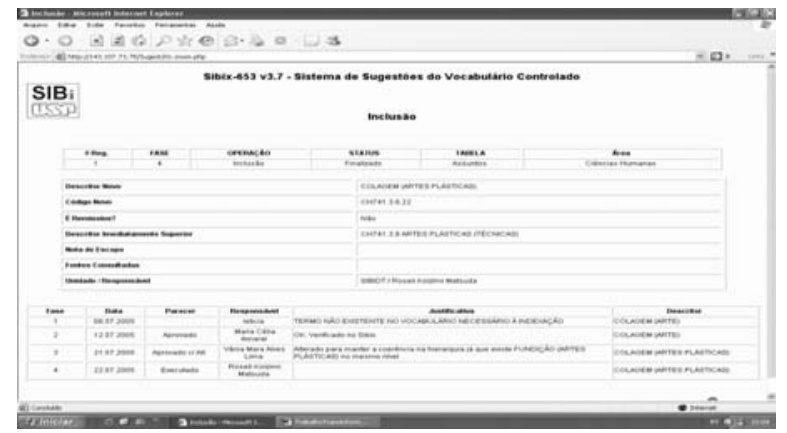

Figura 3. Formulário de Sugestões: solicitação aprovada com alterações.

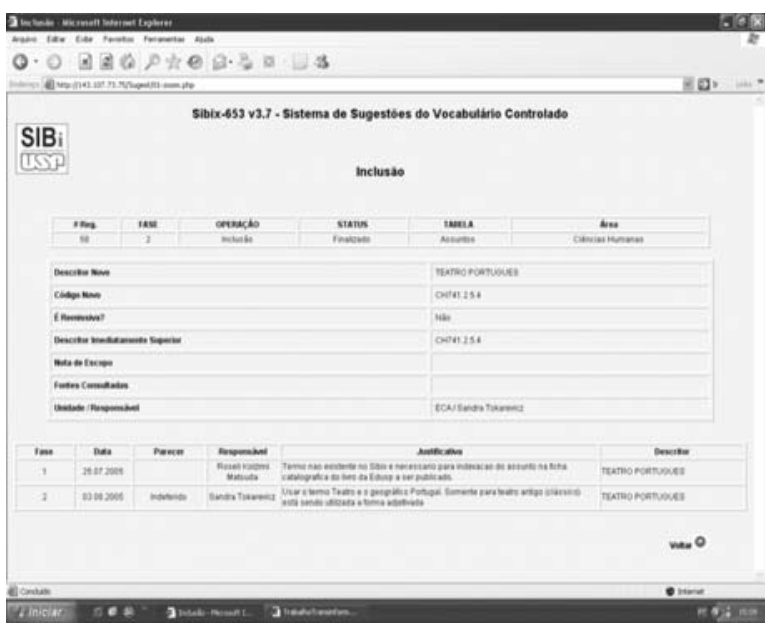

Figura 4. Formulário de Sugestões: solicitação indeferida. 
CONSIDERAÇÕES FINAIS

O "Vocabulário Controlado do SIBi/USP", que se originou de projeto sistêmico, com participação de bibliotecários e especialistas da área acadêmica da USP, é hoje um processo integrado de forma permanente ao Departa-mento Técnico do SIBi/USP. A forma de gerenciamento adotada procura atender às necessidades específicas de trabalhos coletivos em rede. Como se procurou demonstrar, é inerente à construção e manutenção de vocabulários controlados e o seu monitoramento constante. Esse processo requer o uso de instrumentos específicos, baseados em TICs. A estrutura de trabalho adotada, a ferramenta desenvolvida (SIBIX 653) e os fluxos estabelecidos para manutenção do Vocabulário Controlado do SIBi/USP procuraram acompanhar as tendências atuais da área de TICs e de Administração de Sistemas de Informação.

O modelo adotado, que proporciona interação via rede, promove também o melhor aproveitamento dos investimentos efetivados pela Instituição em TICs, conferindo agilidade e visibilidade ao processo de manutenção do Vocabulário, o que agrega valor ao Banco DEDALUS. De fato, o SIBIX 653, expressão material da concepção e execução

\section{REFERÊNCIAS}

BELLUZZO, R.C.B. Liderança \& formação e desenvolvimento de equipes. São Paulo: SIBi/USP, 2003. Apostila.

BENNIS, W.; BIEDERMAN, P.W. Os gênios da organização: as forças que impulsionam a criatividade das equipes de sucesso. 2.ed. Rio de Janeiro: Campus, 1999.

CASTELLS, M. A sociedade em rede. 5.ed. São Paulo: Paze Terra, 2001.

GUINCHAT, C.; MENOU, M. Linguagens documentais. In: GUINCHAT, C. Introdução geral as ciências e técnicas da informação e documentação. Brasília: IBICT, 1994. p.133-165.

KOBASHI, N.Y.; LIMA, V.M.A. Metodologia de desenvolvimento, implantação e gerenciamento do vocabulário controlado da do gerenciamento do Vocabulário Controlado do SIBi/USP, permite aos participantes do Sistema de Bibliotecas propor a atualização do mesmo e acompanhar o processo de análise e validação de suas sugestões, desde a origem até a etapa final, quando se dá a atualização da base de dados do Vocabulário. O esforço empenhado na manutenção da ferramenta temática - Vocabulário Controlado do SIBi/USP - visa, em última instância, proporcionar maior facilidade na recuperação da informação pelo usuário final. Alcança-se, com isso, melhor aproveitamento dos recursos informacionais da Universidade.

A construção de vocabulários controlados, como também sua implantação e geren-ciamento, são tarefas árduas, de longa duração, e que requerem pessoal altamente especializado, bem como recursos financeiros consideráveis. Assim, acredita-se firmemente que esta expe-riência possa ser compartilhada, de modo a contribuir para o aprimoramento dos sistemas de recuperação de informação do País. Destacam-se, ao menos, alguns benefícios da cooperação sugerida: economia de recursos pelo uso responsável de recursos públicos e o compartilhamento de vocabulários controlados com possibilidade de compatibilizar bases de dados de diferentes instituições.

USP. In: SEMINÁRIO NACIONAL DE BIBLIOTECAS UNIVERSITÁRIAS, 13., 2004, Natal. Trabalho apresentado na Mesa redonda: Organização da informação e os instrumentos metodológicos.

SANTOS, C.A.C.M. Linguagens documentarias e codificação da informação: estudo de vocabulário da área da saúde. 2002. 70f. Dissertação (Mestrado em Ciência da Informação) - Escola de Comunicação e Artes, Universidade de São Paulo, São Paulo, 2002.

SVENONIUS, E. The intellectual foundations of information organization. Cambridge: MIT Press, 2001.

TERRA, J.C.C.; GORDON, C. Portais corporativos: a revolução na gestão do conhecimento. São Paulo: Negócio Editora, 2002. 


\section{ANEXO 1}

PROPOSTAPARAGESTÃO DO VOCABULÁRIO

Análise do DT/SIBi - Novembro / 2004

\section{Operações}

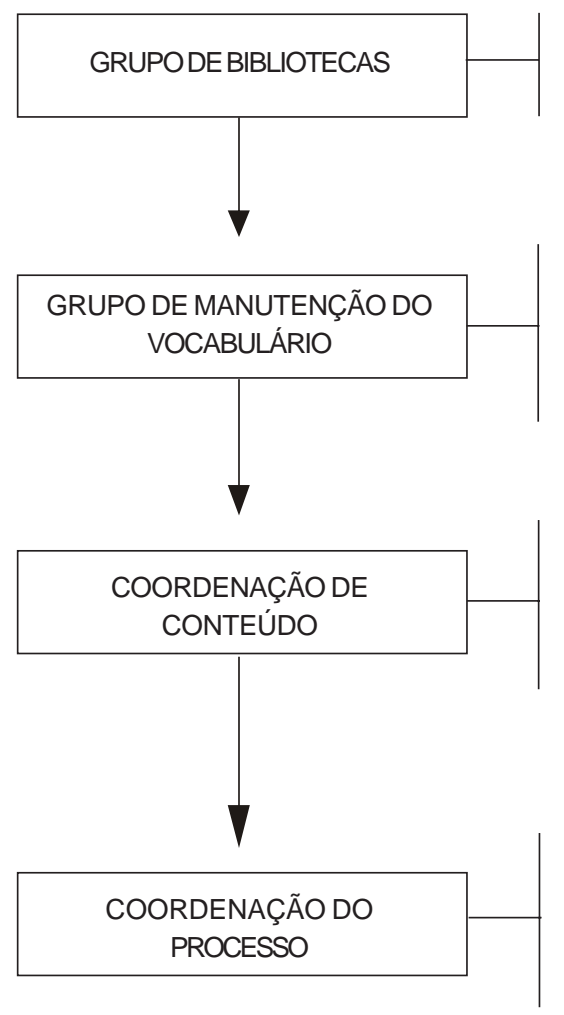

Preenchimento do Formulário

On-line (solicitação/inclusão/ correção/exclusão).

Coleta das sugestões por área; Análise da solicitação;(1) Consulta a especialista, se necessário;(2) Consolidação (confirmação da sugestão); Encaminhamento (deferido/indeferido).

Consistência (definição do descritor na estrutura);

Validação do descritor e da consistência); ${ }^{(3)}$ Regimento do grupo; Política de indexação.

Elaboração de Manuais;

Elaboração de Programas de Treinamento Implementação - inclusão no SIBix;

- alterações no MAN, se necessário.
Instrumentos

\section{Formulário on-line}

\section{Base de Sugestões}

\section{Base deSugestões}

Base de Sugestões

SIBix

MAN

\footnotetext{
1 Grupo composto por 7 bibliotecários, sendo 2 de cada área do conhecimento (sub-coordenadores de área) e 1 coordenador geral.

2 Consultores/professores.

3 Validação da inclusão do termo por especialista (interno ou externo) em vocabulário, tesauro, etc.
} 
UNIVERSIDADE DE SÃO PAULO

SISTEMAINTEGRADODEBIBLIOTECAS

DEPARTAMENTO TÉCNICO

EQUIPE DE GERENCIAMENTO DO VOCABULÁRIO CONTROLADO DO SIBI/USP - 2004-2006

\section{Grupo de Manutenção:}

- Área de Ciências Biológicas:

Vera Regina Casari Boccato (FOB)

Cibele Araújo Camargo Marques dos Santos (FSP)

Suplente: MariaAngela de Toledo Leme (ESALQ)

- Área de Ciências Exatas e Tecnologia:

Silvia Regina Saran Della Torre (EP)

Sonia Regina Yole Guerra (IGc)

Suplente: Marina Mayumi Yamashita (CQ)

- Área de Ciências Humanas:

Maria Célia Amaral (FFLCH)

Sandra Tokarevicz (ECA)

Suplente: Márcia Pilnik (IEB)

Coordenadoria de Conteúdo: Vânia Mara Alves Lima (FAU) e Nair Yumiko Kobashi (Docente ECA)

Coordenadoria de Processo: Mariza L. M. Do Coutto (DT/SIBi)

Grupo de Bibliotecas: formado por bibliotecários indexadores do SIBi/USP, com representação de todas as áreas do conhecimento.

São Paulo, 7 de dezembro de 2004 Departamento Técnico SIBiUSP 
